\title{
Prosthetic rehabilitation of enucleated eye with semi-customized ocular prosthesis: A case report.
}

\author{
Indra Kumar Limbu', Bishal Babu Basnet1, Sandhya Paudel ${ }^{1}$ \\ ${ }^{1}$ Department of Prosthodontics and Crown-bridge, College of Dental Surgery, BP Koirala Institute of \\ Health Sciences, Dharan, Nepal.
}

\section{Correspondence:}

Dr. Indra Kumar Limbu, MDS, Assistant Professor

Department of Prosthodontics and Crown-bridge, College of Dental Surgery,

BP Koirala Institute of Health Sciences, Dharan, Nepal.

E-mail:indralimbulimbu@gmail.com

Article received: June 1, 2019

Article accepted: June 15, 2019

\section{ABSTRACT}

Eye is a vital organ not only for vision but it is also important component of facial expression. The disfigurement associated with loss of eye can cause significant physical and emotional problem. There are several techniques documented in the literature for fabricating the artificial eye. This is a case report of fabricating ocular prosthesis with stock iris and custom made sclera to provide aesthetically satisfactory result and better fit.

Key words: Eye enucleation, Ocular prosthesis, Stock iris.

\section{INTRODUCTION}

Eye is a vital organ not only for vision but it is also important component of facial expression ${ }^{1}$.Surgical removal of eye may be due to trauma,infection,tumour and atrophy of eye. Surgical removal of eye is classified into three categories by Peyman, Saunders and Goldberg ${ }^{2}$ : enucleation, evisceration and exenteration. Enucleation is a surgical procedure in which the globe and the attached portion of the optic nerve are excised from the orbit ${ }^{3}$. Evisceration is removal of the content of the globe leaving the sclera and extra ocular muscles intact. Exenteration involves removal of globe, adnexa and part of an orbit.

The disfigurement associated with loss of eye can cause significant physical and emotional problem ${ }^{4}$. It is very important to immediately rehabilitate such patient with a missing eye. Psychological distress associated with loss of eye can be improved by an ocular prosthesis, simulating natural eye ${ }^{5}$. There are several techniques documented in the literature for fabricating the artificial eye.It can be done by fitting stock eye, modifying stock eye and custom fabrication of eye. Stock eye prosthesis has unreliable fit and is un-aesthetic though it is less time consuming. Custom made eye prosthesis is highly aesthetic and has better fit but it is time consuming and technique sensitive ${ }^{6,7}$. As an alternative, modified stock eye can be made which has advantages of better fit,less time consuming,less technique sensitive and better aesthetic.

This clinical report demonstrate a technique for fabricating ocular prosthesis with stock iris and custom made sclera to provide aesthetically satisfactory result and better fit .

\section{Case description}

A 54-year-old man visited to the Department of Prosthodontics and Crown - Bridge, College of Dental Surgery, BP Koirala Institute of Health Sciences (BPKIHS), Dharan with a chief complaint of missing left eye and desired to wear an artificial eye. He gave the history of penetrating injury to the eye followed by enucleation.

On examination, there was adequate depth between the fornices, which could be an advantage for better retention of the prosthesis (Figure 1). After thorough evaluation and discussion with the patient semi-customized ocular prosthesis was planned to fabricate. 
Figure 1: The patient with enucleated eye.

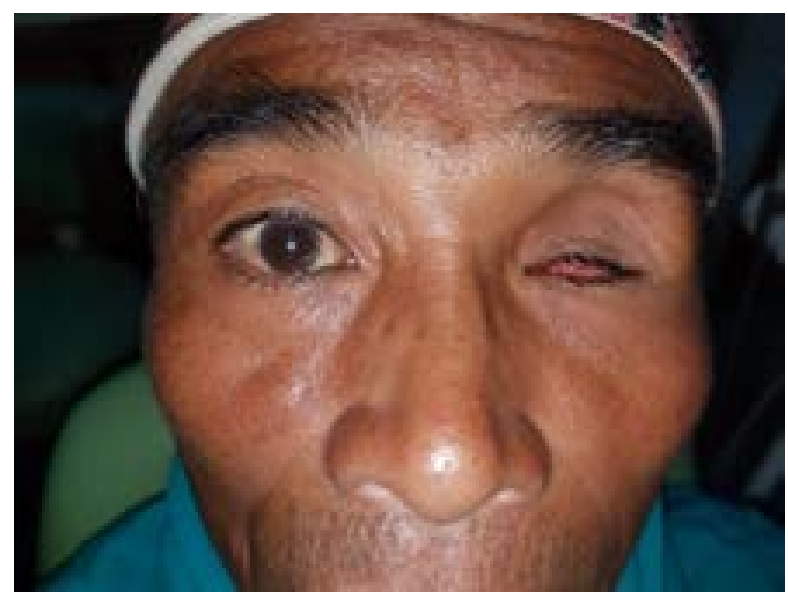

\section{Preliminary Impression of the eye socket}

The impression was made with irreversible hydro colloid, alginate (colto print NCR,Coltene, Switzerland) directly injected by sterile syringe into the eye socket while the patient was gazing forward at a fixed point six feet away. Once set, the impression was removed by having the patient to wiggle his face and blink the eye to break the seal. The impression was removed and inspected for any voids or defects (Figure2). The impression was boxed and poured to ob tain the primary cast.

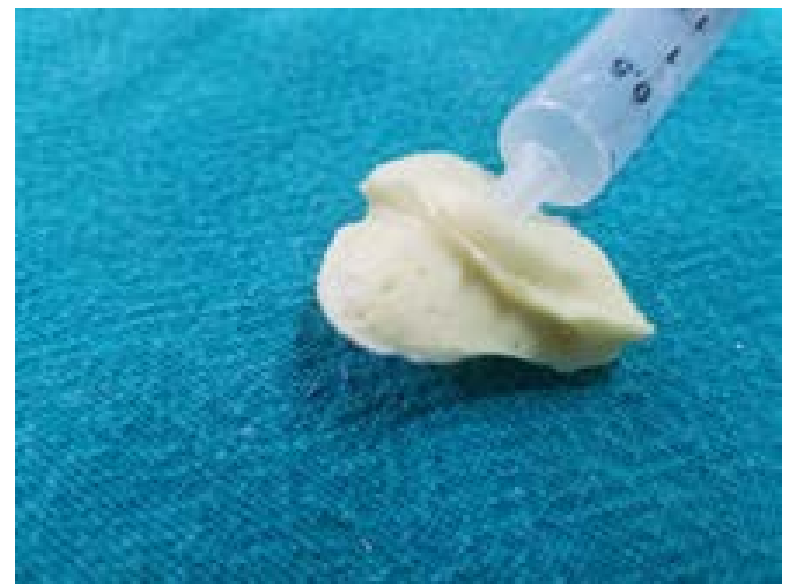

Figure 2: Irreversible hydrocolloid primary impression.

\section{Final impression}

On the obtained primary cast, wax spacer was placed followed by fabrication of custom tray with clearpolymethyl methacrylate (DPI RR cold cure, India) using sprinkle-on technique.A hollow tube was attached at the center of the custom tray for injecting the secondary impression materialand relief holes were made (Figure 3, a).Light body polyvinyl siloxane impression material (Affinis,Coltene,Switzerland) was then injected through the tube attached to the special tray into the eye socket and the material was allowed to set. The impression was removed from the eye socket (Figure 3, b) and poured with dye stone(Kalrock,Kalabhai,India)to obtain split cast mold.

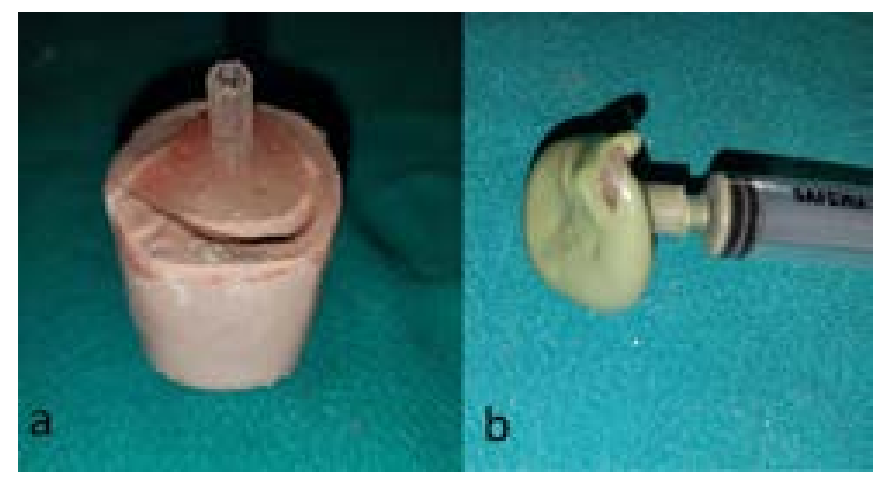

Figure 3, a: The custom tray, b: The final impression with light body impression material.

\section{Wax pattern fabrication and try- in}

The mold was coated by a layer of separating media. Molten modeling wax (DPI modeling wax, Dental Product of India, India) was poured into mold.Once the wax was solidified, wax pattern was retrieved by splitting the mold. The fit of the pattern was checked in the patient by observing the extension of the wax pattern into the fornices (Figure 4). The areas of over extensions or under extensions were adjusted by removing or adding the wax.

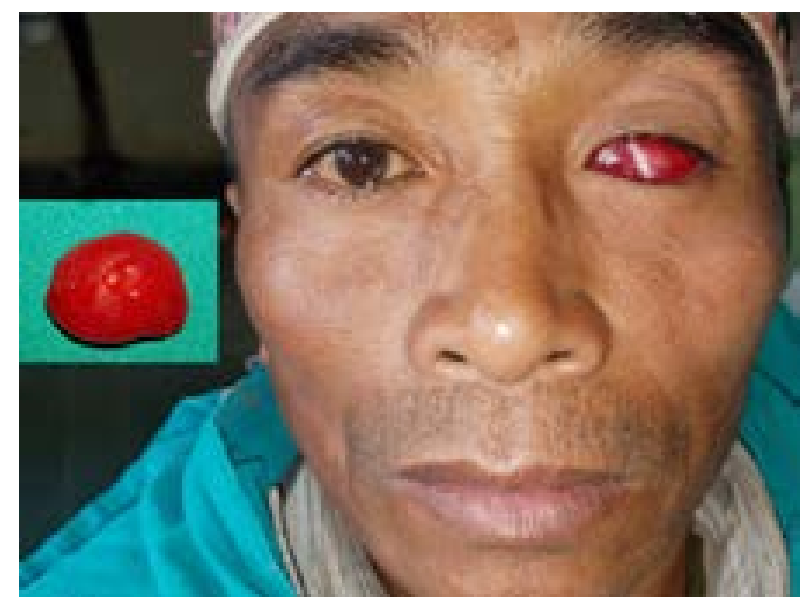

Figure 4: Wax pattern try-in. The inset shows wax pattern outside eye socket.

\section{Fabrication of resin sclera and try-in}

After satisfactory fit, contour, and support of the pattern, it was flasked and processed (Figure 5,a,b) with heat cure acrylic resin(DPI-Heat cure, India)The processed scleral blank was polished and checked into the socket for fit and comfort.Adjustments required were carried out until the patient could close his eyelid comfortably with the scleral blank in position (Figure 6). 


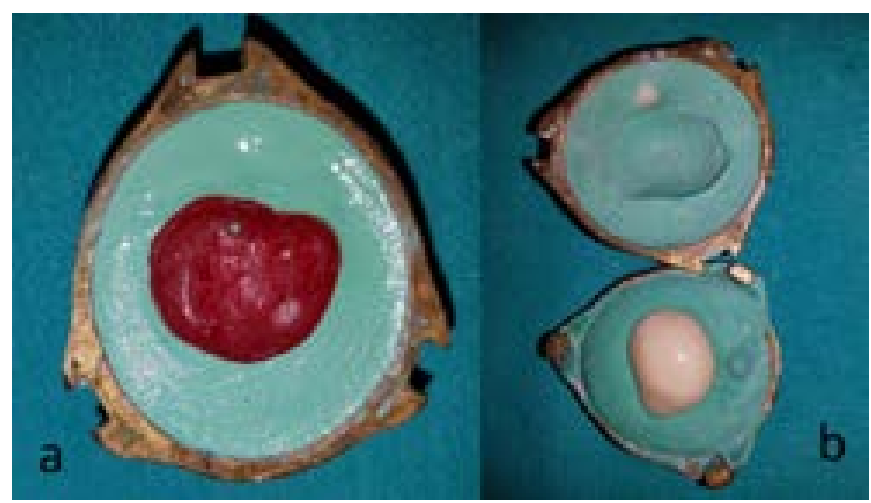

Figure 5, a: Investing the wax pattern. b: The processed scleral blank.

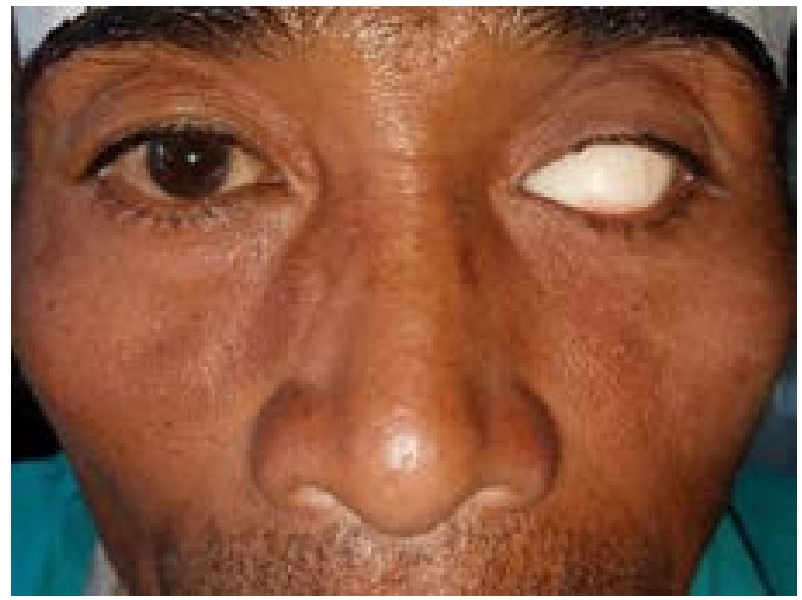

Figure 6: Scleral blank try-in; marking position of iris.

\section{Selection and positioning of the iris}

The size, shade and position of the iris were selected keeping the natural eye as a guide. Properly matched iris was selected from the commercially available stock eye. The position of the iris was determined by asking the patient to look ahead at a distant object. In the natural eye, the distance from medial canthus to the medial border of iris was measured with the help of divider. Similarly, the distance from lateral canthus to the lateral border of iris was measured.These markings were then transferred to the scleral blank to get the exact position of the iris while the patient looked at distant object (figure 6). The area marked for iris was then reduced with an acrylic trimming bur to create a space for iris. The trimmed iris obtained from stock eye was adapted in the trimmed scleral blank with the help of modeling wax. It was then tried in. The patient was asked to look left and right, upwards and downwards to perform various eye movements. The harmony of movements between the natural eye and the scleral-iris assembly was examined and verified.

\section{Characterization and final processing of the prosthesis}

An abrasive stone was used to reduce $1 \mathrm{~mm}$ of the anterior surface of the scleral blank over which the wax was coated to make space for the clear acrylic layer. An acrylic sprue was attached to the iris for proper positioning of it during dewaxing and processing. Then the assembly was flasked and dewaxing was done. The space left by the wax was now filled with clear acrylic resin.Fine red threads were placed on the scleral portion to mimic the blood vessels. The entire scleral portion was coated with a thin layer of clear acrylic syrup to keep the blood vessel fibers in place. Once set, the scleral blank was replaced on to the flask and the processing was done. After completion of the processing, it was polished using sandpaper followed by polishing cake and buff(Figure 7, in situ).

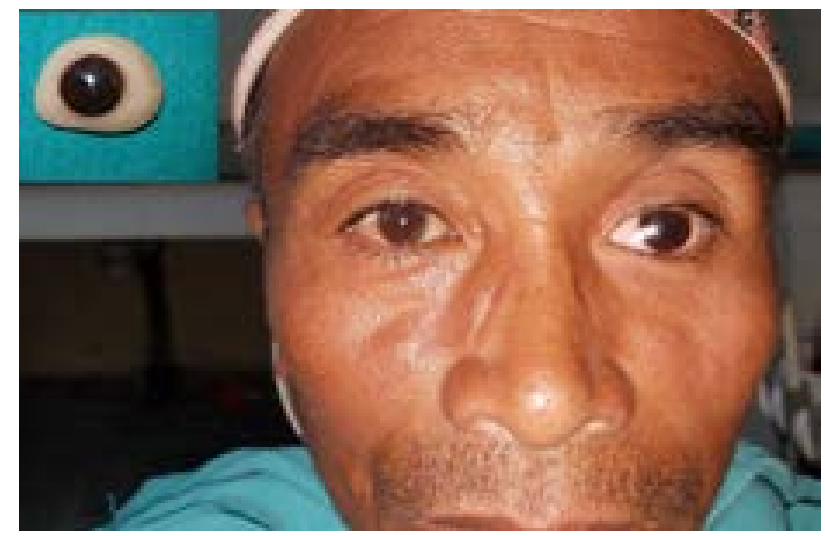

Figure 7: The prosthesis in-situ. The inset shows finished and polished prosthesis.

\section{Insertion and delivery of final prosthesis}

Finished prosthesis was inserted and checked for fit, contour, and movements (Figure7). Esthetics and harmonious movement with the other eye were compared. Necessary adjustments were made.Postinsertion instruction was given to the patient regarding usage,limitations and maintenance of prosthesis. He was advised to wear protective glasses to protect the natural eye as well as to camouflage the prosthesis with natural eye.

\section{DISCUSSION}

Custom made ocular prosthesis provides better results functionally as well as aesthetically ${ }^{8}$. But fabricating iris by painting the scleral disc takes more time and needs artistic skill. Using the stock iris and custom fabricated 
sclera has its ownadvantage. Selecting a properly matched iris, trimming it and incorporating it in custom made sclera takes less time.For this, meticulous painting skill is not needed. On the other hand,directly fitting stock eye into eye socket makes patient uncomfortable and because of improper fit of stock eye, there maybe gaps accumulating the secretions but eye prosthesis fabricated by this method has better fit and no secretion accumulation. The procedure described in this report is an easy technique where custom made scleral fitting the eye socket is fabricated beforehand and an iris is obtained readily from stock prosthesis. However, in this technique there are some disadvantages. We cannot fabricate eye prosthesis by this technique if proper matching iris is not available.Since we need to do the flasking and curing procedure two times, it is time consuming.

Different ocular impression techniques had been discussed by various authors. Allen and Webster 9 recommended using perforated stock ocular tray for alginate impression. Doshi and Aruna recommended directly injecting impression material into the eye socket ${ }^{8}$. Impression making without fabricating custom tray and directly injecting material into socket has improper support for impression obtained.Custom ocular tray impression with its proper support has better detail so it helps in proper adaptation. We described the method to fabricate a tray before making the final impression.

To make eye prosthesis very realistic, mobility of eye ball is important. Solution for this is by using ocular implants. There are various types of ocular implants available ${ }^{10,11}$.Placing implants is complicated surgical procedure and can be done only by ocular surgeon. Moreover it is an expensive procedure and patients with low economic statuscannot afford.Acrylic resin ocular prosthesis is a viable alternative for such patients ${ }^{12}$.

\section{CONCLUSION}

This semi customized technique reduces laboratory time and does not demand the artistic skills whereas it provides satisfactory aesthetics and better fit.

\section{REFERENCES}

1. Mishra SK, Choudhary R. Reproduction of custom made eye prosthesis maneuver: a case report. J Dentist Oral Hygiene. 2009: 1; 59-63.
2. Peyman GA, Sanders DR, Goldberg MF. Principles and practice of ophthalmology. New Delhi: Jaypee Brothers Medical Brothers (P) Ltd. 1987: 233-4

3. Scoll DB. The Anophthalmic socket.Ophthalmol. 1982:89; 407-23.

4. Lubkin V, Sloan S.Enucleation and psychic trauma. Advances in Ophthalmic Plastic and Reconstructive Surgery.1990:8; 259-62.

5. Putanikar NY, Patil AG, Shetty PK,Nagaral S,Mithaiwala HI. Prosthetic rehabilitation of a patient with ocular defect using semi-customized prosthesis: A case report, J Int Oral Health. 2015:7; 81-4.

6. Benson P. The fitting and fabrication of a custom resin artificial eye. J Prosthet Dent. 1977:38; 532-8.

7. Taicher S, Steinberg HM, Tubiana I, Sela M. Modified stockeye ocular prosthesis. J Prosthet Dent. 1985: 54; 95-8.

8. Doshi PJ, Aruna B :Prosthetic management of Soc. 2005:5; 37-8.

9. Allen I, Webster HE :Modified impression method of artificial eye fitting. Am. J. Ophthalmol.1969: 67: 189-218.

10. Gouglemann HP.The evolution of ocular motility implant.In:Oculoplasstic surgery and prosthetics, Shannon GM,Connely FJ,eds.Boston: Little,Brown. (1970) 689-711.

11. YousefYA.Enucleation surgery -orbital implants and surgical technique.US ophthalmic review 2016:9; 46-8.

12. Somkumar K,Mathai R,Jose P. Ocular prosthesis patient rehabilitation: A case report. People's J Scientific Res. 2009:2; 21-6. 